\title{
Natalizumab in Early Relapsing-Remitting Multiple Sclerosis: A 4-Year, Open-Label Study
}

\author{
Jai Perumal $\cdot$ Roumen Balabanov $\cdot$ Ray $\mathrm{Su} \cdot$ Roger Chang \\ Laura Balcer · Steven Galetta • Denise I. Campagnolo • \\ Robin Avila $\cdot$ Lily Lee $\cdot$ Danette Rutledge $\cdot$ Robert J. Fox
}

Received: January 26, 2021 / Accepted: March 22, 2021 / Published online: May 20, 2021

(c) The Author(s) 2021, corrected publication 2021

\section{ABSTRACT}

Introduction: STRIVE was a 4-year, multicenter, observational, open-label, single-arm study of natalizumab treatment in anti-JC virus antibody-negative (JCV-negative) relapsing-remitting multiple sclerosis (RRMS) patients with disease duration $\leq 3$ years. The objective of STRIVE was to examine no evidence of disease activity (NEDA) status and predictors of NEDA in natalizumab-treated patients with early RRMS.

Drs. Su and Lee were affiliated with Biogen (Cambridge, MA, USA) at the time of this study.

Supplementary Information The online version contains supplementary material available at https:// doi.org/10.1007/s12325-021-01722-w.

J. Perumal $(\bowtie)$

Department of Neurology and Neuroscience, Weill

Cornell Medical College, Cornell University, 1300

York Avenue, New York, NY 10065, USA

e-mail: jaisperumal@gmail.com

R. Balabanov

Department of Neurology, Northwestern University,

Chicago, IL, USA

R. Su

Inari Medical, Irvine, CA, USA

R. Chang - D. I. Campagnolo - R. Avila · D. Rutledge

Biogen, Cambridge, MA, USA
Methods: Proportions of patients with NEDA were evaluated along with baseline predictors of NEDA, annualized relapse rate, 24-week confirmed disability worsening (CDW), magnetic resonance imaging assessments (T2 and gadolinium-enhancing lesions), and serious adverse events.

Results: In years 1 and 2, 56.1\% (95\% confidence interval [CI] 48.7-63.4\%) and $73.6 \%$ (95\% CI $66.2-80.2 \%$ ) of patients (intent-to-treat population $[N=222])$, respectively, achieved NEDA. In years 3 and $4,84.6 \%(95 \%$ CI 78.0-89.9\%) and 91.9\% (95\% CI 86.4-95.8\%) of patients, respectively, achieved Clinical NEDA (no relapses or 24-week CDW). Baseline predictors of NEDA in year 4 were Expanded Disability Status Scale score $\leq 2.0$ (odds ratio $[\mathrm{OR}]=3.85$ [95\% CI 1.54-9.63]; $p=0.004)$ and T2 lesion

\section{Balcer · S. Galetta}

Department of Neurology, New York University

School of Medicine, New York, NY, USA

L. Lee

TG Therapeutics, New York, NY, USA

R. J. Fox

Mellen Center for Multiple Sclerosis, Cleveland Clinic, Cleveland, $\mathrm{OH}$, USA 
volume $>4 \mathrm{cc}(\mathrm{OR}=0.39$ [95\% CI $0.15-0.98]$; $p=0.046)$, with the latter also predicting Clinical NEDA in year $4(\mathrm{OR}=0.21[95 \%$ CI $0.05-0.92] ; p=0.038$ ). The cumulative probability of CDW at year 4 was $19.3 \%$. Serious adverse events were reported in $11.3 \%$ of patients.

Conclusion: These results support the longterm safety and effectiveness of natalizumab. Baseline predictors of NEDA help to inform benefit-risk assessments of natalizumab treatment in JCV-negative patients with early RRMS.

Trial Registration: ClinicalTrials.gov identifier NCT01485003.

Keywords: Anti-JCV antibody negative; Magnetic resonance imaging; Natalizumab; No evidence of disease activity; Relapsing-remitting multiple sclerosis

\section{Key Summary Points}

Why carry out this study?

Natalizumab treatment was previously shown to significantly reduce disease activity in patients with relapsingremitting multiple sclerosis (RRMS), which led to the development of a new outcome measure called no evidence of disease activity (NEDA; no relapses or confirmed disability worsening [CDW], no gadolinium-enhancing lesions, and no new or newly enlarging T2 lesions).

To inform benefit-risk assessments of natalizumab treatment in anti-JC virus (JCV) antibody-negative patients with early RRMS, this analysis of the STRIVE study evaluated achievement of NEDA and Clinical NEDA (no relapses or CDW), baseline predictors of NEDA and Clinical NEDA, and effectiveness and safety outcomes.

\section{What was learned from the study?}

In STRIVE, $56.1 \%$ and $73.6 \%$ of patients achieved NEDA in years 1 and 2, respectively, and $84.6 \%$ and $91.9 \%$ of patients achieved Clinical NEDA in years 3 and 4 , respectively.

In addition to achievement of NEDA and Clinical NEDA, the cumulative probability of CDW at year 4 was $19.3 \%$, and safety outcomes were consistent with the established safety profile of natalizumab, supporting the long-term effectiveness and safety of natalizumab.

This study also identified baseline predictors of NEDA in year 4 (Expanded Disability Status Scale score $\leq 2.0$ and T2 lesion volume $>4 \mathrm{cc}$ ) and Clinical NEDA in year 4 (baseline T2 lesion volume $>4$ cc), which may help to inform benefit-risk assessments of natalizumab treatment in anti-JCV antibody negative patients with early RRMS.

\section{DIGITAL FEATURES}

This article is published with digital features, including a summary slide, to facilitate understanding of the article. To view digital features for this article go to https://doi.org/10.6084/ m9.figshare.14248406.

\section{INTRODUCTION}

Natalizumab significantly reduced clinical and magnetic resonance imaging (MRI) disease activity in relapsing-remitting multiple sclerosis (RRMS) patients compared with placebo over 2 years in the AFFIRM trial [1]. These findings prompted a post hoc analysis and the subsequent development of a new outcome measure called no evidence of disease activity (NEDA; no relapses or confirmed disability worsening [CDW], no gadolinium-enhancing [Gd+] lesions, and no new or newly enlarging T2 lesions) [2]. 
Natalizumab treatment is associated with a risk of progressive multifocal leukoencephalopathy (PML), an opportunistic viral infection of the brain caused by the JC virus (JCV) [3, 4]. As infection with JCV is required for the development of PML [3], this study only enrolled patients who were anti-JCV antibody negative (JCV negative), since the estimated PML risk in these patients is $1 / 10,000$, irrespective of treatment duration or prior immunosuppressant use $[5,6]$. Therefore, they have the most favorable benefit-risk profile of all natalizumab-treated patients with multiple sclerosis (MS).

The study rationale was to evaluate NEDA and its predictors to inform benefit-risk assessments of natalizumab treatment in JCV-negative patients with early RRMS. The primary study objective was to determine the impact of natalizumab treatment on achieving NEDA in years 1 and 2 and no relapses or 24-week CDW (Clinical NEDA) in years 3 and 4. Secondary objectives included baseline predictors of NEDA and Clinical NEDA as well as annualized relapse rate (ARR), 24-week CDW, MRI assessments, and serious adverse events (SAEs).

\section{METHODS}

\section{Study Design and Patients}

STRIVE (Study of Tysabri in early RelapsingremItting multiple sclerosis in anti-JCV antibody negatiVE patients; clinicaltrials.gov NCT01485003) was a prospective, 4-year, observational, open-label, multicenter phase 4 study conducted at 45 sites in the USA. The first patient was treated in February 2012, and the study ended in November 2018. Patients received $300 \mathrm{mg}$ of natalizumab intravenously every 4 weeks.

Eligible patients were RRMS (McDonald 2010 criteria) [7] patients aged 18-65 who were JCV negative within 6 months prior to screening or at the baseline visit and had a disease duration $\leq 3$ years and an Expanded Disability Status Scale (EDSS) score $\leq 4.0$. Each patient was naive to disease-modifying therapies (DMTs) or had been treated with a DMT for $\leq 36$ months prior
Table 1 Inclusion and exclusion criteria

Inclusion criteria

Aged 18-65 years at the time of informed consent

Documented diagnosis of relapsing-remitting MS

(McDonald 2010 criteria) [7]

Disease duration of $\leq 3$ years from diagnosis at the time of informed consent

EDSS score $\leq 4.0$

Tested negative for anti-JCV antibodies $\leq 6$ months prior to screening or at the baseline visit

Satisfied the therapeutic indications for natalizumab

Treatment naive to DMT or treated with

DMT(s) (including but not limited to intramuscular interferon beta-1a, subcutaneous interferon beta-1a or beta- $1 \mathrm{~b}$, glatiramer acetate, or fingolimod)

for $\leq 3$ years in total prior to the informed consent date

Decision made to treat with natalizumab preceded enrollment

Ability to understand the purpose and risks of the study and provide signed and dated informed consent and authorization to use protected health information in accordance with national and local patient privacy regulations

Exclusion criteria

Prior natalizumab treatment

Anti-JCV antibody positive at any time point prior to or at baseline

Contraindications to treatment with natalizumab

History of opportunistic infections or an increased risk for such infections

Primary progressive or secondary progressive MS

History of immunosuppressant use (e.g., mitoxantrone, azathioprine, methotrexate, cyclophosphamide, mycophenolate, cladribine, or rituximab)

Immunocompromised at the time of enrollment 
Table 1 continued

Known active malignancies, with the exception of cutaneous basal cell carcinoma that had been completely excised prior to study entry

Women breastfeeding, pregnant, or planning to become pregnant, or not postmenopausal or surgically sterile but unwilling to practice contraception

Inability to comply with study requirements

$D M T$ disease-modifying therapy, EDSS Expanded Disability Status Scale, JCV JC virus, $M S$ multiple sclerosis

to the date of informed consent. Table 1 provides a complete list of inclusion and exclusion criteria. Patients' decision to initiate natalizumab treatment was made prior to enrollment.

All patients provided written informed consent prior to enrollment. Approval was granted by the Copernicus Group IRB \#1 (reference number IRB00001313) and, at the rest of the study sites, by an independent ethics committee (Supplementary Appendix S1). The study was performed in accordance with the Helsinki Declaration of 1964 and its later amendments.

\section{Assessments}

Demographic and clinical characteristics were recorded at the screening visit. Relapses were assessed at baseline and at follow-up clinic visits every 6 months ( \pm 1 month) [8]. EDSS assessments and anti-JCV antibody tests $[6,9]$ were conducted at the screening visit and every 6 months ( \pm 1 month) from baseline. Patients who had an anti-JCV antibody-positive test result during the course of the study could continue on natalizumab at the discretion of the treating neurologist.

MRI was conducted at baseline and yearly thereafter and analyzed by a central reader, NeuroRx Research (Montreal, Quebec, Canada). A standardized MRI protocol and quantitative image analysis were used in this study. The pulse sequences included proton densityweighted, T2-weighted, and T1-weighted scans acquired pre-gadolinium injection; a fluid-attenuated inversion-recovery scan immediately post-gadolinium injection; and a T1-weighted scan post-gadolinium injection. All scans for each subject were performed on the same scanner and head coil throughout the study. Images were quantitatively analyzed by the central MRI reading center to calculate total T2 lesion volume, Gd+ lesion count, and new or newly enlarging T2 lesion count using the center's automated image analysis pipeline followed by expert review to remove any falsepositive lesions.

Twenty-four-week CDW was defined as a $\geq 1.5$-point increase from a baseline EDSS score of $0.0, \mathrm{a} \geq 1.0$-point increase from a baseline EDSS score of 1.0 to $<6.0$, or $a \geq 0.5$-point increase from a baseline score $\geq 6.0$, confirmed 24 weeks later.

NEDA included attainment of both Clinical NEDA, defined as no relapses and no 24-week CDW, and MRI NEDA, defined as no Gd+ lesions and no new or newly enlarging $\mathrm{T} 2$ lesions.

Results based on additional secondary outcome measures collected in STRIVE, including confirmed disability improvement, retinal nerve fiber layer thickness, low- and high-contrast visual acuity, cognitive impairment, capacity for work, and quality of life, will be published separately.

Any SAE that occurred between the time of informed consent and the end of the study was reported by the participating neurologist or designee as soon as possible, preferably within $24 \mathrm{~h}$ of his/her becoming aware of the event.

If a patient permanently discontinued natalizumab treatment but chose to remain in the study, data were collected on the reasons for discontinuation. In such cases, the investigative site continued to follow-up with the patient as per the protocol schedule of assessments through month 48. If a patient withdrew from the study, the participating neurologist documented the reason for early withdrawal on the study exit form and conducted the final assessments, after which no further data were collected. 


\section{Endpoints}

The primary study endpoints were the proportion of patients achieving NEDA in years 1 and 2 and Clinical NEDA in years 3 and 4 . Secondary endpoints reported in this article included baseline characteristics that predicted NEDA and Clinical NEDA in year 4 as well as ARR, 24-week CDW, MRI measures (Gd+ and new or newly enlarging T2 lesions), and SAEs.

\section{Statistical Analysis}

Analyses were conducted in the intent-to-treat (ITT) population, defined as all enrolled patients who completed informed consent and received $\geq 1$ dose of natalizumab in STRIVE.

To account for potential disease activity shortly after natalizumab initiation, an exploratory analysis assessed NEDA over years 2-4 following MRI rebaselining at year 1 . Patients with missing data who did not exhibit disease activity on the available measurements were excluded from the analysis of NEDA, whereas patients missing data who had disease activity on $\geq 1$ measurement were considered not to have achieved NEDA.

In general, continuous variables were analyzed using summary statistics, and categorical variables were analyzed using frequency distributions. Analyses were conducted using twosided tests with a type I error rate of 0.05. Multivariate logistic regression tested the association between baseline characteristics, including age, sex, MS disease duration, prior MS treatment history, relapses in the prior year, EDSS score, Gd+ lesions, T2 lesion volume, and NEDA and Clinical NEDA status in year 4 . These baseline characteristics were considered potential confounders for each other. A confounder was included if it changed any coefficients of other covariates in the model. ARRs were analyzed with a repeated Poisson model, and cumulative probabilities of CDW were estimated using the Kaplan-Meier method. To assess the robustness of the results and any potential attrition bias linked to treatment effectiveness, sensitivity analyses on NEDA, Clinical NEDA, MRI NEDA, ARR, CDW, and
MRI were performed using the data from all patients who completed the 4-year study on natalizumab. The total number and incidence of SAEs were estimated based on the proportion of patients experiencing $\geq 1$ SAE in the ITT population.

\section{Data Availability Statement}

The datasets generated and/or analyzed during the current study are not publicly available. The authors and company are fully supportive of allowing independent assessment and verification of these results. Requests for de-identified data should be made via the established company data-sharing policies and processes detailed on the website http://clinicalresearch. biogen.com/.

\section{RESULTS}

\section{Patient Enrollment and Baseline Characteristics}

Table 2 summarizes the baseline characteristics of the ITT population $(n=222)$. Patients had active disease at baseline, as indicated by a Gd+ lesion count ranging from 0 to 71 (median $=0$ ) and a median (range) EDSS score of $2.0(0-6.5)$, as well as a median (range) of $1(0-12)$ relapses in the year before natalizumab treatment initiation. Half of the patients in the ITT population had a history of MS treatment, the majority of whom were previously on injectables (i.e., any interferon or glatiramer acetate). Baseline characteristics for patients who completed all 4 years of the study on natalizumab treatment (termed "4-year natalizumab completers"; $n=105$ ) were similar to those of the ITT population (Table 2).

Patient enrollment and disposition data are shown in Fig. 1.

Overall, 91 of 222 patients (41.0\%) discontinued natalizumab (Table 3) and 67 of 222 patients $(30.2 \%)$ withdrew from the study (Fig. 1). The top reason for natalizumab discontinuation was seroconversion to anti-JCV antibody-positive status/elevated index/PML risk $(n=27)$. Reported lack of efficacy was listed 
Table 2 Baseline characteristics of the intent-to-treat (ITT) population and of patients who completed 4 years of natalizumab treatment

\begin{tabular}{|c|c|c|}
\hline Baseline characteristic & $\begin{array}{l}\text { ITT population } \\
(n=222)\end{array}$ & $\begin{array}{l}\text { 4-Year natalizumab completers } \\
(n=105)\end{array}$ \\
\hline Age, mean (SD), years & $34.0(9.0)$ & $34.1(8.9)$ \\
\hline Female, $n(\%)$ & $161(72.5)$ & $74(70.5)$ \\
\hline \multicolumn{3}{|l|}{ Race and ethnicity, $n(\%)^{\mathrm{a}}$} \\
\hline White, not Hispanic or Latino & $152(68.5)$ & $71(67.6)$ \\
\hline Black or African American & $40(18.0)$ & $24(22.9)$ \\
\hline Hispanic or Latino & $18(8.1)$ & $6(6.3)$ \\
\hline Asian, American Indian, or Alaska Native & $3(1.4)$ & $2(1.9)$ \\
\hline Time from diagnosis of MS, mean (SD), years & $1.6(0.8)$ & $1.62(0.8)$ \\
\hline Time since MS symptom onset, mean (SD), years & $3.0(2.6)$ & $2.83(2.3)$ \\
\hline \multicolumn{3}{|l|}{ Number of relapses in the past year } \\
\hline Mean $(S D)$ & $1.4(1.2)$ & $1.43(0.9)$ \\
\hline Median (range) & $1(0-12)$ & $1.00(0-4)$ \\
\hline \multicolumn{3}{|l|}{ EDSS score } \\
\hline Mean (SD) & $2.0(1.1)$ & $2.0(1.0)$ \\
\hline Median (range) & $2.0(0-6.5)$ & $2.0(0-4.0)$ \\
\hline T2 lesion volume, median (range), cc & $3.9(0-61.6)^{b}$ & $4.5(0-61.6)^{c}$ \\
\hline Number of Gd+ lesions, median (range) & $0(0-71)^{\mathrm{b}}$ & $0(0-71)^{c}$ \\
\hline Patients with no Gd+ lesions, $n(\%)$ & $114(57.9)^{\mathrm{b}}$ & $50(51.6)^{c}$ \\
\hline MS treatment history, $n(\%)^{\mathrm{d}}$ & $111(50.0)$ & $45(42.9)$ \\
\hline Any interferon & $54(48.6)$ & $27(60.0)$ \\
\hline Intramuscular interferon beta-1a & $29(26.1)$ & $12(26.7)$ \\
\hline Subcutaneous interferon beta- $1 \mathrm{~b}$ & $10(9.0)$ & $7(15.6)$ \\
\hline Subcutaneous interferon beta- $1 \mathrm{a}$ & $19(17.1)$ & $8(17.8)$ \\
\hline Glatiramer acetate & $50(45.0)$ & $17(37.8)$ \\
\hline Dimethyl fumarate & $7(6.3)$ & $3(6.7)$ \\
\hline Prednisolone & $7(6.3)$ & $0(0.0)$ \\
\hline IV methylprednisolone & $6(5.4)$ & $2(4.4)$ \\
\hline Teriflunomide & $3(2.7)$ & $1(2.2)$ \\
\hline Methylprednisolone & $3(2.7)$ & $0(0.0)$ \\
\hline Fingolimod & $1(0.9)$ & $0(0.0)$ \\
\hline Medrol dose pack & $1(0.9)$ & $0(0.0)$ \\
\hline \multicolumn{3}{|c|}{ Duration of MS treatment in those with history of use, weeks } \\
\hline Mean (SD) & $37.4(29.1)$ & $40.45(33.4)$ \\
\hline
\end{tabular}


Table 2 continued

\begin{tabular}{lll}
\hline Baseline characteristic & $\begin{array}{l}\text { ITT population } \\
(\boldsymbol{n}=\mathbf{2 2 2})\end{array}$ & $\begin{array}{l}\text { 4-Year natalizumab completers } \\
(\boldsymbol{n}=\mathbf{1 0 5})\end{array}$ \\
\hline Median (min, max) & $30.6(0.4,165.4)$ & $31.4(1.1,165.4)$ \\
\hline$D M T$ disease-modifying therapy, EDSS Expanded Disability Status Scale, $G d+$ gadolinium enhancing, $I V$ intravenous, max \\
maximum, min minimum, $M S$ multiple sclerosis, $S D$ standard deviation \\
a Data were missing for 9 of 222 patients $(4.1 \%)$ \\
b $n=197$ \\
c $n=97$ \\
d Patients may have reported prior use of $>1$ treatment
\end{tabular}

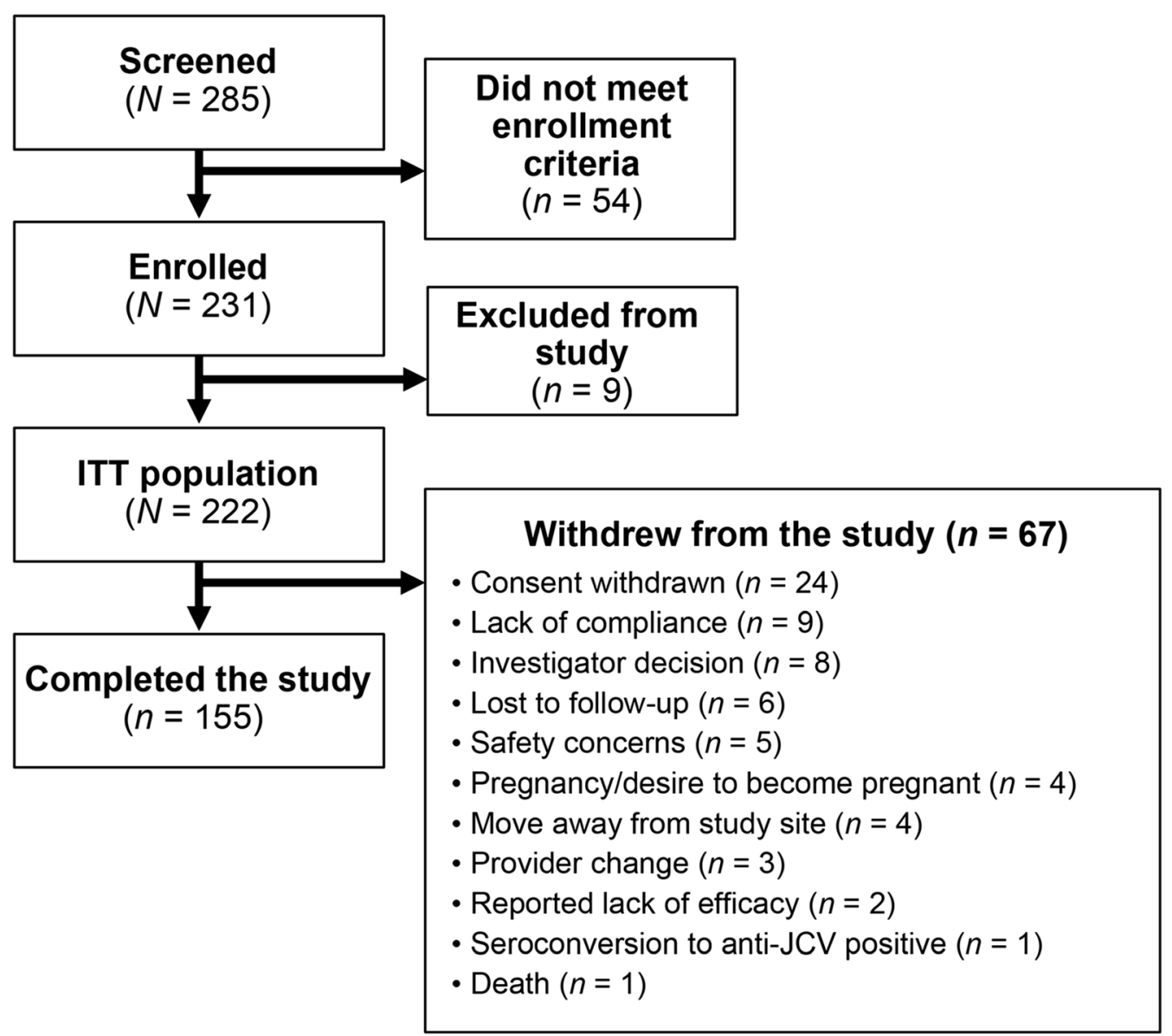

Fig. 1 Patient disposition. JCV JC virus ITT intent to treat

as a reason for discontinuation of natalizumab in only eight patients (Table 3). The top reason for study withdrawal was withdrawal of consent $(n=24)$.
At screening, anti-JCV antibody status was known for 200 of the 222 patients included in the ITT population (Fig. 2). As patients with a positive test result were excluded, all 200 patients with a known antibody status were JCV 
Table 3 Reasons for natalizumab discontinuation in the intent-to-treat population

\begin{tabular}{|c|c|}
\hline $\begin{array}{l}\text { Reason for natalizumab discontinuation, } \\
n(\%)\end{array}$ & $N=222$ \\
\hline Any reason & $91(41.0)$ \\
\hline Investigator decision & $7(3.2)$ \\
\hline Consent withdrawn & $11(5.0)$ \\
\hline Compliance & $10(4.5)$ \\
\hline Pregnancy/desire to become pregnant & $7(3.2)$ \\
\hline Adverse event & $10(4.5)$ \\
\hline Reported lack of efficacy & $8(3.6)$ \\
\hline $\begin{array}{l}\text { Seroconversion to anti-JCV positive status/ } \\
\text { elevated index/PML risk }\end{array}$ & $27(12.2)$ \\
\hline Lost to follow-up & $3(1.4)$ \\
\hline Insurance change & $3(1.4)$ \\
\hline Anti-natalizumab antibodies & $2(0.9)$ \\
\hline Death & $1(0.5)$ \\
\hline Unknown/other & $2(0.9)$ \\
\hline
\end{tabular}

negative at screening. Of the 22 patients who did not have anti-JCV antibody testing at screening, 4 had unknown test results throughout the remainder of the study, 9 had a negative test result at month 6 , and 5 had a negative test result at month 12 . One patient who did not have a test result at screening or month 6 tested positive for anti-JCV antibodies at month 12. Another patient who did not have a test result at screening, month 6 , or month 12 tested negative for anti-JCV antibodies at month 18. Finally, two patients did not have test results until month 48; both were JCV negative at that time. Of the four patients with unknown test results throughout the remainder of the study, all were considered eligible for inclusion in the study based on a negative antiJCV antibody status within 6 months prior to screening. For those patients in whom anti-JCV antibody results were known at month 6 and subsequent time points, the percentage who remained JCV negative decreased from $88.8 \%$ at month 6 to $77.9 \%$ at month 48 .

\section{NEDA}

In the ITT population, $56.1 \%$ and $73.6 \%$ of patients achieved NEDA in years 1 and 2, respectively; $84.6 \%$ and $91.9 \%$ of patients achieved Clinical NEDA in years 3 and 4, respectively (Fig. 3). The exploratory analysis with MRI rebaselining at year 1 found that $70.1 \%$ of patients achieved Clinical NEDA, $83.7 \%$ achieved MRI NEDA, and $58.0 \%$ of patients achieved NEDA in years 2-4 (Fig. 3). Similar results were observed in the 4-year natalizumab completers.

Among the baseline characteristics evaluated for predictors of NEDA (age, sex, MS disease duration, MS treatment history, relapse in the prior year, EDSS score, Gd+ lesions, and T2 lesion volume), T2 lesion volume $>4 \mathrm{cc}$ was significantly associated with Clinical NEDA in year 4 (Fig. 4). Baseline T2 lesion volume $>4 \mathrm{cc}$ was also significantly associated with NEDA in year 4, as was baseline EDSS score $\leq 2.0$ (Fig. 4).

Exploratory analyses showed that achievement of NEDA in year 4 was not significantly associated with Clinical NEDA, MRI NEDA, or NEDA status in year 1 (Fig. 5). Of the patients who had disease activity in year $1(n=82)$, $64.6 \%$ exhibited new or newly enlarging T2 lesions, whereas relapses, 24-week CDW, and $\mathrm{Gd}+$ lesions were each seen in $\leq 28.0 \%$ of patients (Table 4). Among patients with disease activity in years $2(n=43), 3(n=42)$, and $4(n=$ 35 ), $41.9 \%, 47.6 \%$, and $62.9 \%$, respectively, had new or newly enlarging $\mathrm{T} 2$ lesions in those years (Table 4). Similarly, an exploratory analysis of achievement of NEDA over years 2-4 based on NEDA status in year 1 indicated that the likelihood of achieving NEDA over years 2-4 was generally similar irrespective of whether NEDA was achieved in year 1 (data not shown).

\section{Clinical and MRI Outcomes}

In the ITT population, ARR decreased by $90.0 \%$, from $1.41 \quad(95 \%$ confidence interval $[\mathrm{CI}]$ : 


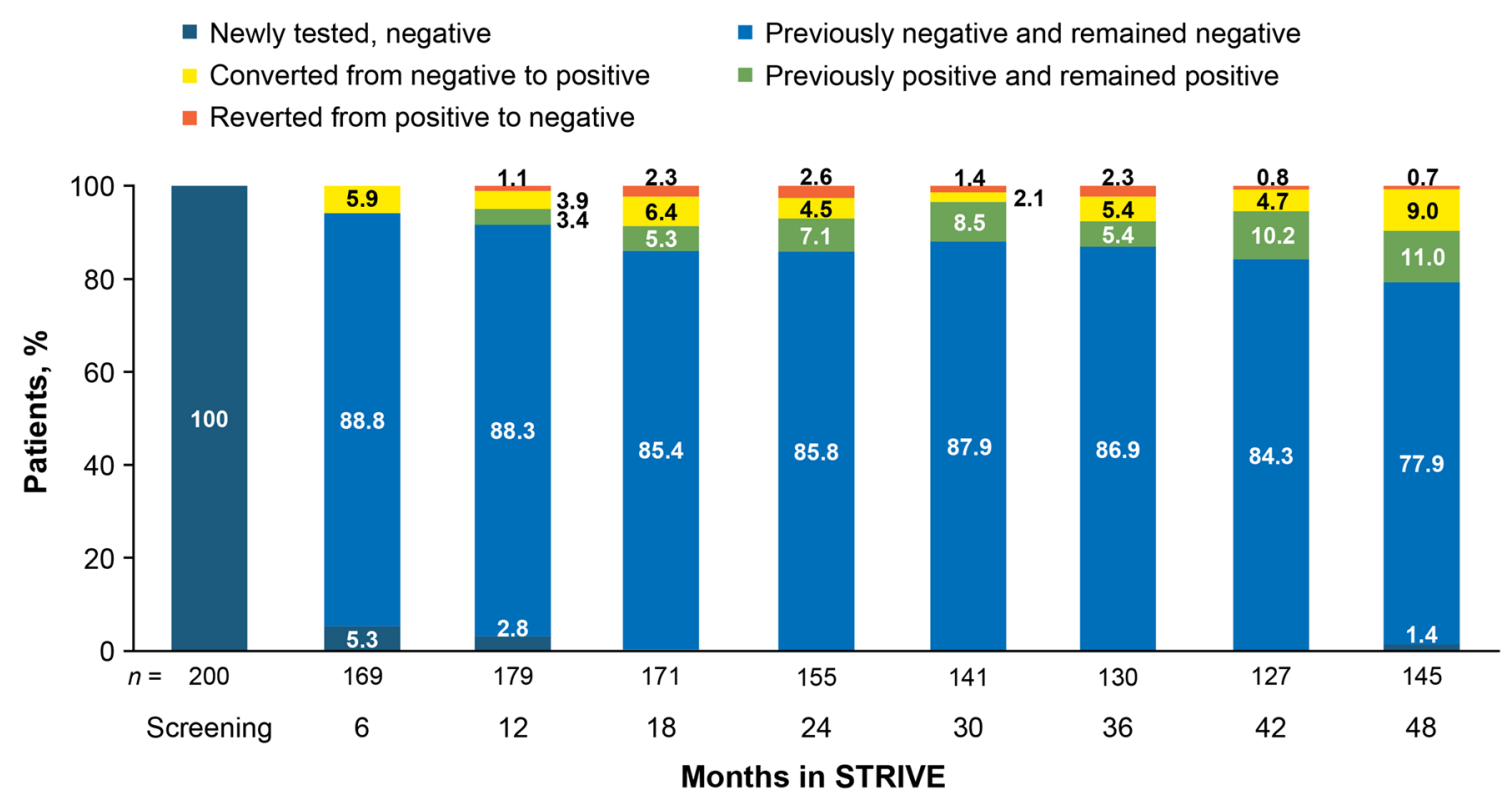

Fig. 2 Anti-JC virus antibody status in the intent-to-treat population by change from previously available test result (regardless of whether the patient remained on natalizumab treatment or not)

1.19-1.66) in the year prior to natalizumab to 0.14 (95\% CI 0.10-0.21) at the end of the study $(p<0.0001$; Fig. 6a). At year 4 , the cumulative probability of 24 -week CDW was $19.3 \%$ (Fig. 6b). Similar results were observed in the 4-year natalizumab completers, with an ARR of 0.12 and a cumulative probability of 24 -week CDW of $17.3 \%$ at year 4 . The mean (standard deviation) EDSS score at year 4 was 1.77 (1.55) and 1.78 (1.54) for the ITT population and the 4-year natalizumab completers, respectively.

In the ITT population, in each year, the mean number of new or newly enlarging T2 lesions was $\leq 1.02$ and the median number of new or newly enlarging T2 lesions was 0 , with a range of $0-23$ in year 1 and $0-41$ in year 4 . In each year, the mean number of $\mathrm{Gd}+$ lesions was $\leq 0.07$ and the median number of $\mathrm{Gd}+$ lesions was 0 , with a range of $0-3$ in year 1 and $0-2$ in year 4 (Table 5). The reduction from baseline in the number of $\mathrm{Gd}+$ lesions was significant in years $1-4(p<0.0001$ for all). Similar results were observed in the 4-year natalizumab completers.

\section{Safety}

SAEs were reported in 25 of 222 patients (11.3\%; Table 6), with no cases of PML. SAEs reported in $\geq 2$ patients included MS relapse $(n=5 ; 2.3 \%)$, intervertebral disc protrusion $(n=2 ; 0.9 \%)$, and suicide attempt $(n=2 ; 0.9 \%)$. One patient had a treatment-related $\mathrm{SAE}$, an anaphylactic reaction, but was not hospitalized for evaluation. One patient had an SAE leading to death. The cause of death was reported as "traumatic brain injury, subdural hematoma" and was not considered by the investigator to be treatment related. The patient had received only one dose of natalizumab, and the death was reported $>3$ months after natalizumab administration.

\section{DISCUSSION}

In this 4-year study of natalizumab-treated patients with early RRMS, the primary study objective was to determine the impact of natalizumab treatment on achieving NEDA in years 1 and 2 and Clinical NEDA in years 3 and 4. The results showed that $56.1 \%$ and $73.6 \%$ of patients achieved NEDA in years 1 and 2, 

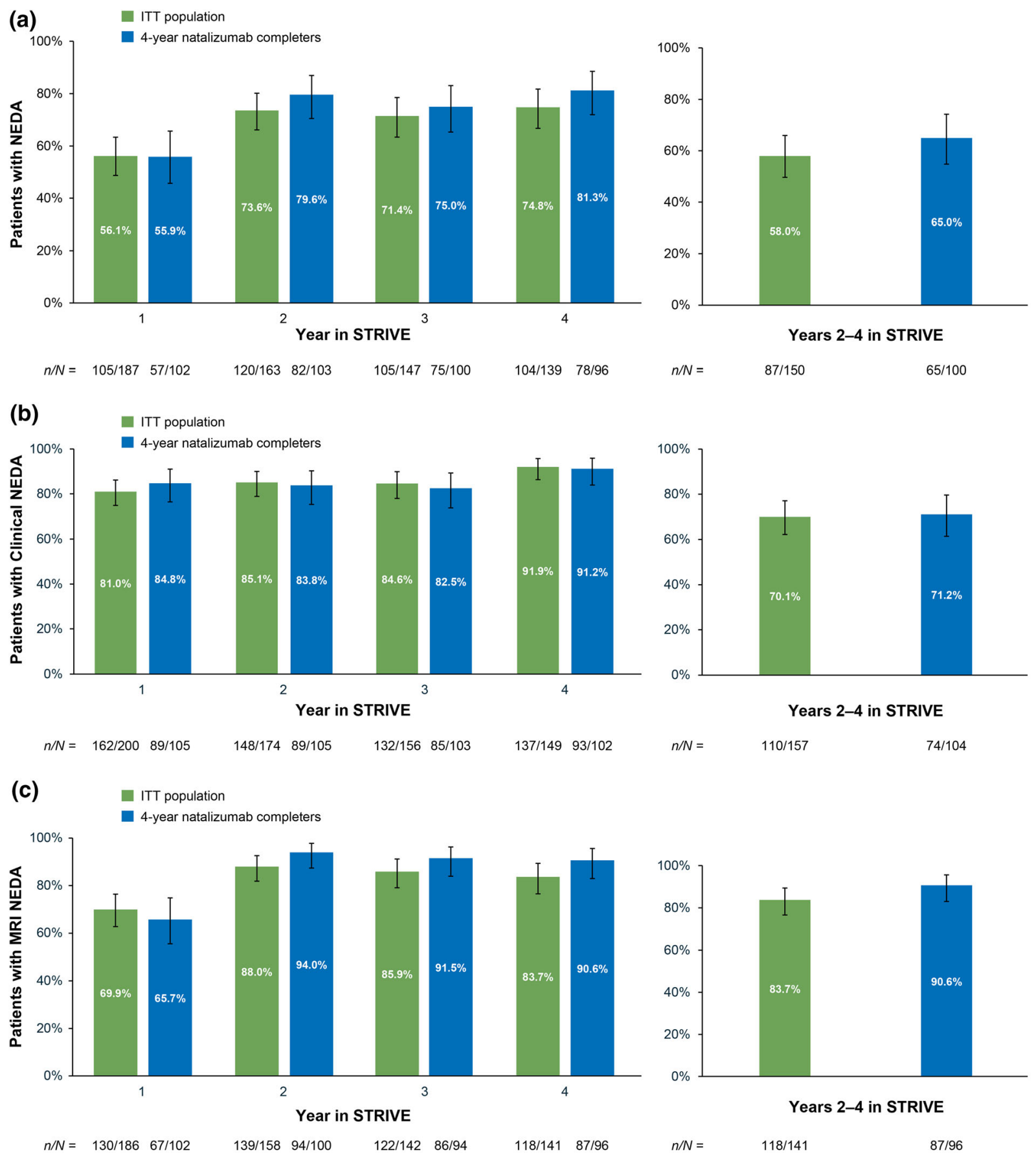

Fig. 3 a No evidence of disease activity (NEDA), $\mathbf{b}$ clinical NEDA, and $\mathbf{c}$ MRI NEDA in each year of STRIVE and in years 2-4 of STRIVE with rebaselining in the STRIVE intent-to-treat (ITT) and 4-year natalizumab completer

respectively, and $84.6 \%$ and $91.9 \%$ of patients achieved Clinical NEDA in years 3 and 4, respectively. An increase in the rate of NEDA

populations. Clinical NEDA no relapses or 24-week confirmed disability worsening, MRI NEDA no gadolinium-enhancing lesions and no new or newly enlarging T2hyperintense lesions

after the first year of natalizumab treatment has previously been reported; in AFFIRM, the proportion of patients achieving NEDA increased 
(a) Covariate

Age ( $<40$ years vs $\geq 40$ years)

MS disease duration ( $\leq 1$ year vs $>1$ year)

Baseline EDSS score ( $\leq 2.0$ vs $>2.0)$

No. of relapses in year prior to starting natalizumab ( $\leq 1$ vs $>1$ )

No. of $\mathrm{Gd}+$ lesions at baseline ( 0 vs $\geq 1$ )

Volume of T2 lesions at baseline ( $\leq 4 \mathrm{cc}$ vs $>4 \mathrm{cc}$ )

MS treatment history (no vs yes)
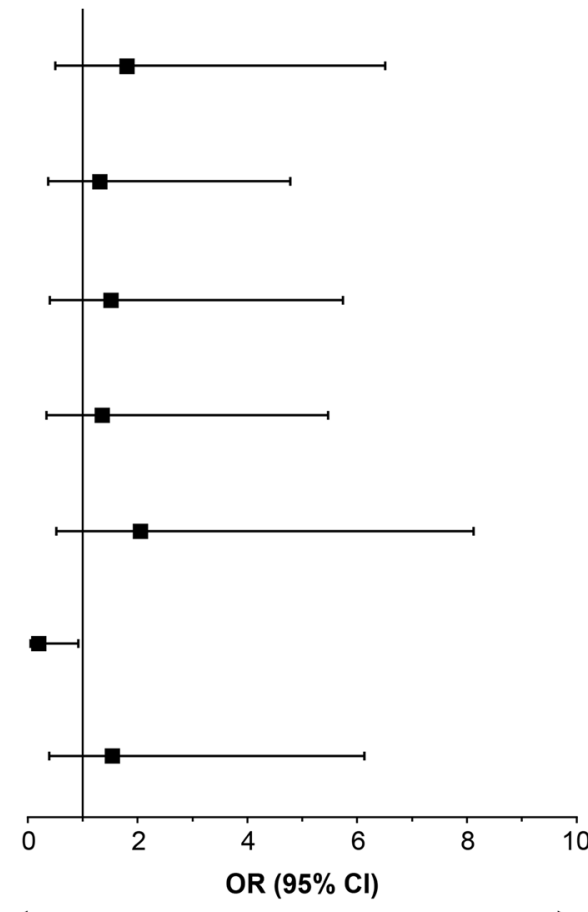

NEDA less likely NEDA more likely

\section{(b) Covariate}

Age ( $<40$ years $v s ~ \geq 40$ years)

Sex (female vs male)

MS disease duration ( $\leq 1$ year vs $>1$ year)

\section{Baseline EDSS score ( $\leq 2.0$ vs $>2.0)$}

No. of relapses in year prior to starting natalizumab ( $\leq 1$ vs $>1)$

No. of $\mathrm{Gd}+$ lesions at baseline ( $0 \mathrm{vs} \geq 1$ )

Volume of T2 lesions at baseline ( $\leq 4 \mathrm{cc}$ vs $>4 \mathrm{cc}$ )

MS treatment history (no vs yes)

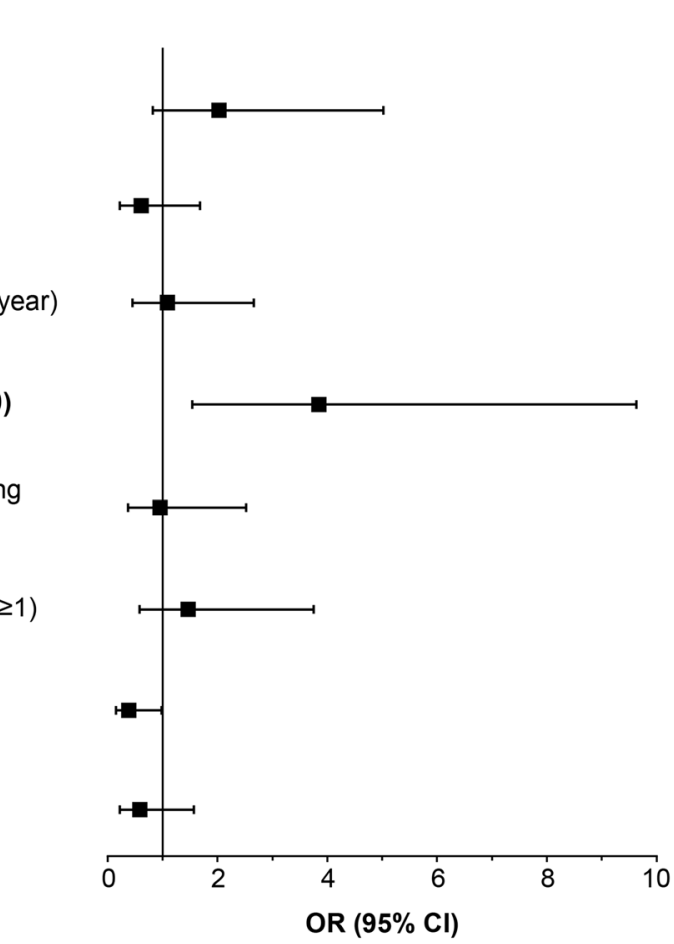

OR $(95 \% \mathrm{Cl})$

$2.03(0.82-5.02)$

$0.61(0.22-1.68)$

$1.09(0.45-2.66)$

$3.85(1.54-9.63)$

$0.96(0.37-2.52)$

$1.47(0.58-3.75)$

$0.39(0.15-0.98)$

$0.59(0.22-1.57)$ $p$ value

0.363

0.668

0.537

0.669

0.303

0.038

0.534

0.124

0.339

0.847

0.004

0.931

0.422

0.046

0.290 
4 Fig. 4 Baseline characteristics that predicted a clinical NEDA and $\mathbf{b}$ NEDA in the intent-to-treat ITT population at year 4 of STRIVE. Odd ratios (ORs) are based on multivariate logistic regression. Statistically significant predictors $(p<0.05)$ are shown in bold. CI confidence interval, Clinical NEDA no relapses or 24-week confirmed disability worsening, EDSS Expanded Disability Status Scale, $G d+$ gadolinium enhancing, $M S$ multiple sclerosis, $N E D A$ no evidence of disease activity

from $47 \%$ in year 1 to $68 \%$ in year 2 [2]. In year 1 of STRIVE, which was similar to AFFIRM [2], a smaller proportion of patients achieved MRI NEDA than Clinical NEDA. Furthermore, in patients with disease activity in year $1(n=82)$, $64.6 \%$ exhibited new or newly enlarging T2 lesions. Thus, MRI disease activity before or shortly after natalizumab initiation may have contributed to a lower proportion of patients achieving NEDA early in STRIVE. However, the percentage of patients who achieved NEDA in year 4 did not differ significantly by Clinical NEDA, MRI NEDA, or NEDA status in year 1, indicating that any disease activity in the first year of STRIVE did not impact the likelihood of NEDA later on.

In addition to annual assessments, the cumulative proportion of patients with NEDA in years 2-4 was analyzed. Due to potential disease activity prior to the onset of action of any given DMT, clinical guidance recommends conducting a brain MRI approximately 6 months after switching to another DMT to establish a new baseline [10]. In STRIVE, MRIs were conducted on an annual basis and, therefore, re-baselining for this analysis was conducted at 1 year. Generally consistent with the annual rates, this exploratory analysis demonstrated that $70.1 \%$ of patients achieved Clinical NEDA, 83.7\% achieved MRI NEDA, and 58.0\% of patients achieved NEDA in years 2-4 with rebaselining at year 1 .

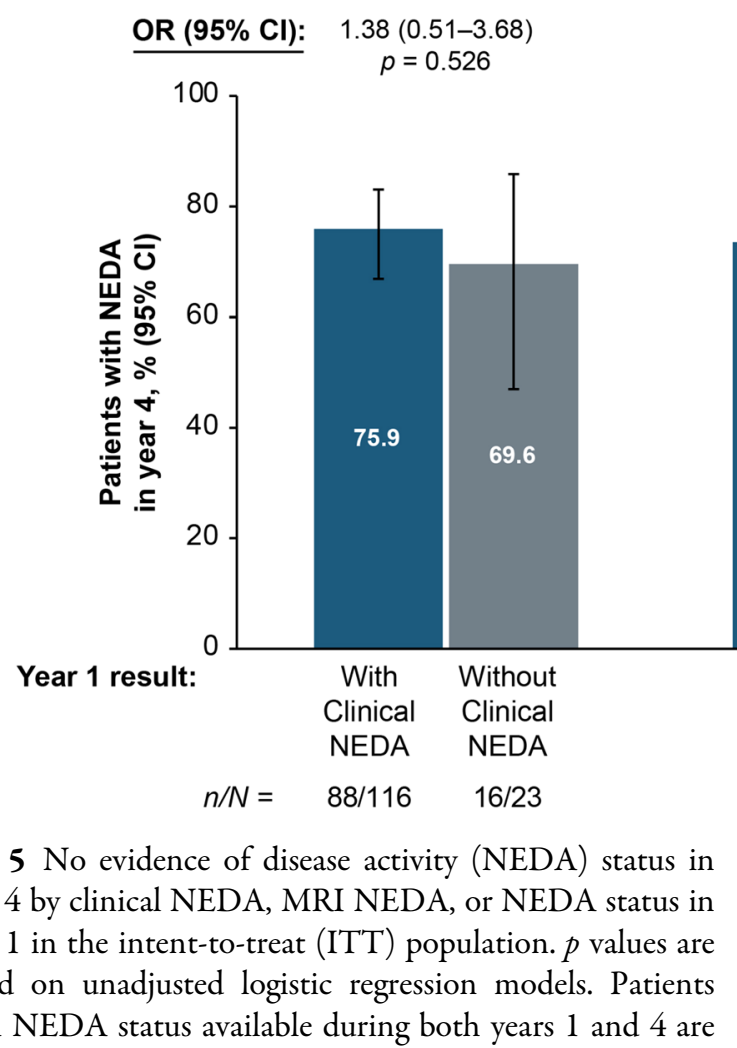

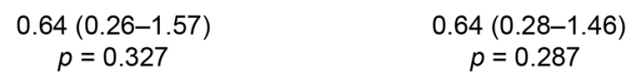

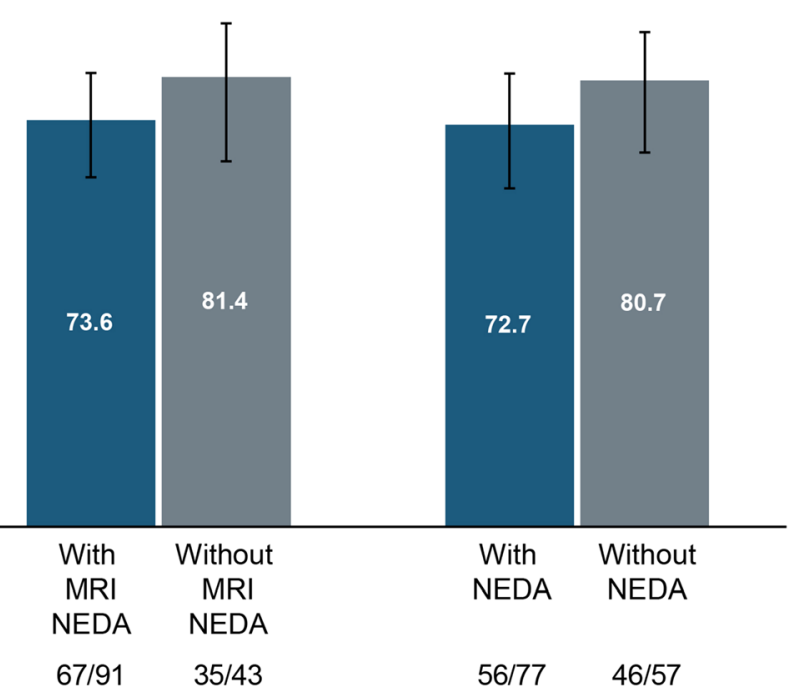

included. $C I$ confidence interval, Clinical NEDA no relapses or 24-week confirmed disability worsening, $M R I$ $N E D A$ no gadolinium-enhancing lesions and no new or newly enlarging T2-hyperintense lesions, $O R$ odds ratio with NEDA status available during both years 1 and 4 are 
Table 4 Patients with disease activity in the intent-to-treat (ITT) population

\begin{tabular}{|c|c|c|c|c|}
\hline & $\begin{array}{l}\text { Year } 1 \\
(n=187)\end{array}$ & $\begin{array}{l}\text { Year } 2 \\
(n=163)\end{array}$ & $\begin{array}{l}\text { Year } 3 \\
(n=147)\end{array}$ & $\begin{array}{l}\text { Year } 4 \\
(n=139)\end{array}$ \\
\hline Patients with disease activity, $n(\%)^{\mathrm{a}}$ & $82(43.8 \%)$ & $43(26.4 \%)^{c}$ & $42(28.6 \%)^{\mathrm{c}}$ & $35(25.2 \%)^{c}$ \\
\hline Patients who relapsed, $n(\%)^{\mathrm{b}}$ & $23(28.0 \%)$ & $20(46.5 \%)$ & $15(35.7 \%)$ & $11(31.4 \%)$ \\
\hline Patients with CDW, $n(\%)^{\mathrm{b}}$ & $19(23.2 \%)$ & $8(18.6 \%)$ & $10(23.8 \%)$ & $2(5.7 \%)$ \\
\hline Patients with $\geq 1 \mathrm{Gd}+$ lesion, $n(\%)^{\mathrm{b}}$ & $8(9.8 \%)$ & $6(14.0 \%)$ & $4(9.5 \%)$ & $9(25.7 \%)$ \\
\hline Patients with $\geq 1$ new or enlarging T2 lesion, $n(\%)^{\mathrm{b}}$ & $53(64.6 \%)$ & $18(41.9 \%)$ & $20(47.6 \%)$ & $22(62.9 \%)$ \\
\hline
\end{tabular}

One of the main secondary objectives of STRIVE was to identify which baseline characteristics, if any, could predict NEDA and Clinical NEDA in year 4 . The results indicated that baseline EDSS score $\leq 2.0$ and T2 lesion volume $>4$ cc were significant predictors of NEDA in year 4 , with the latter also predicting Clinical NEDA in year 4 . A lower baseline EDSS score was previously shown to be associated with a greater chance of maintaining NEDA in natalizumabtreated relapsing MS patients at 7 years [11]. STRIVE, however, is the first study to identify baseline T2 lesion volume $>4 \mathrm{cc}$ as a significant predictor of long-term (i.e., 4-year) NEDA and Clinical NEDA in natalizumab-treated patients. One potential reason for this association may be natalizumab's mechanism of action. Natalizumab reversibly disrupts the interaction between an a4-integrin, expressed on the surface of all leukocytes with the exception of neutrophils, and its receptors, suppressing the formation of Gd+ lesions after the first infusion and allowing for a fast onset of action in relapsing MS patients [12]. The underlying pathology of T2 lesions in MS patients is known to be nonspecific and may reflect varying degrees of inflammation, demyelination, gliosis, edema, and tissue loss [13]. However, as STRIVE enrolled RRMS patients within 3 years of their diagnosis, extensive tissue loss would not be expected. Therefore, one potential hypothesis is that natalizumab may allow time for the T2 lesions, which reflect overall burden of disease, to resolve and potentially even repair in early RRMS patients, with the effect likely to be most evident in those with a high T2 lesion burden at baseline. Further studies are needed to confirm the association between baseline $\mathrm{T} 2$ lesion volume and long-term NEDA.

Consistent with the high (> 80\%) annual rates of Clinical NEDA in STRIVE, the results of the secondary objectives evaluating ARR and 24-week CDW showed low levels of clinical disease activity. In particular, the ARR decreased by $90.1 \%$, and the cumulative probability of 24 -week CDW at 4 years was also low (19.3\%). Similar results were found in the interim analysis of the real-world Tysabri Observational Program (TOP), in which the ARR decreased by $84.9 \%$, from 1.99 the year before treatment initiation to 0.30 at 1 year of treatment, remaining low $(<0.31)$ through 5 years, and with a $16 \%$ cumulative probability of 24 -week CDW at 5 years [14]. Similarly low levels of MRI disease activity, evidenced by the fact that $83.7 \%$ of patients achieved MRI NEDA following MRI rebaselining at year 1 , are also supported by results of the secondary objective evaluating annual MRI outcomes. The median number of $\mathrm{Gd}+$ lesions and new or newly enlarging T2 lesions at each annual assessment was 0 , indicating that over half of the patients did not exhibit new radiological activity. Safety outcomes in STRIVE were consistent with the 
(a)

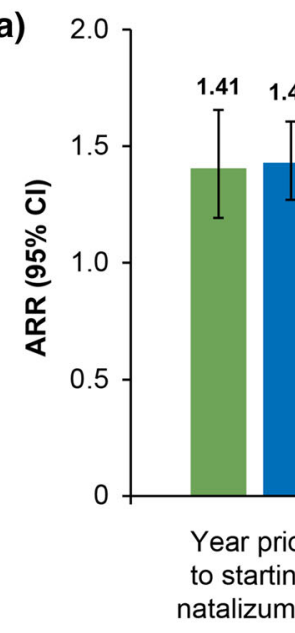

ITT population

4-year natalizumab completers

(b)

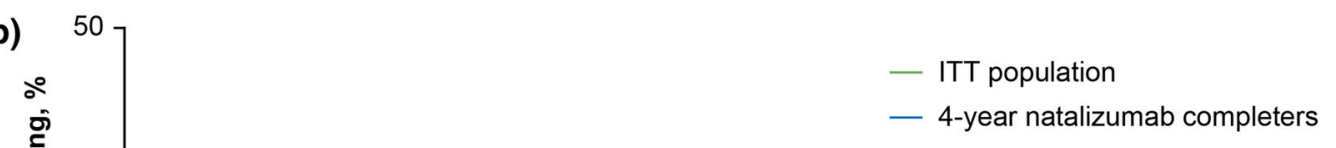

40

ڤั

至

30

은

눈

章 20

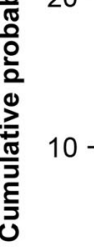

ITT population 222

4-year natalizumab 105 completers

Fig. 6 Summary of the clinical outcome measures a annualized relapse rate (ARR) and b 24-week confirmed disability worsening over 4 years in STRIVE in the

well-established safety profile of natalizumab $[1,5,15,16]$, and there were no cases of PML. The rate of natalizumab discontinuation in intent-to-treat (ITT) and 4-year natalizumab completer populations. Dashed lines indicate $95 \%$ confidence interval (CI)

STRIVE (41.0\%) was less than that reported in other observational studies of MS patients treated with natalizumab $(47.4-52.2 \%)[15,16]$. 


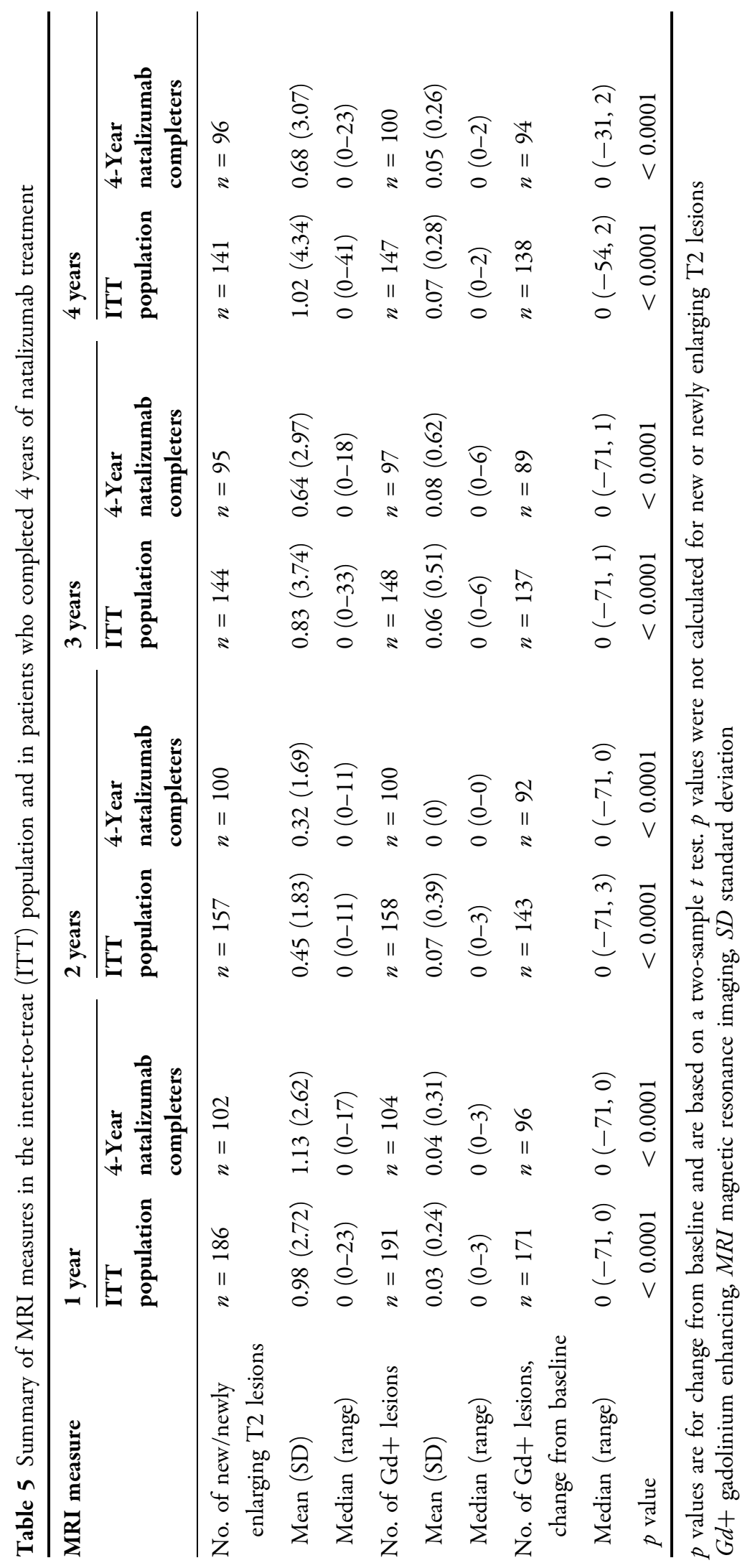


Table 6 Summary of serious adverse events (SAEs) over 4 years in the intent-to-treat population

\begin{tabular}{lc}
\hline Category, $\boldsymbol{n}(\%)$ & $\boldsymbol{N}=\mathbf{2 2 2}$ \\
\hline Patients with $\geq 1$ SAE & $25(11.3)$ \\
SAEs reported in $\geq 2$ patients, MedDRA preferred term, $n(\%)^{\mathrm{a}}$ & \\
MS relapse & $5(2.3)$ \\
Intervertebral disc protrusion & $2(0.9)$ \\
Suicide attempt & $2(0.9)$ \\
Patients with $\geq 1$ treatment-related SAE & $1(0.5)^{\mathrm{b}}$ \\
Patients with $\geq 1$ SAE leading to death & $1(0.5)^{\mathrm{c}}$ \\
\hline
\end{tabular}

SAEs that occurred from the first dose of natalizumab through 30 days after the last dose are included

MedDRA Medical Dictionary for Regulatory Activities, $M S$ multiple sclerosis

${ }^{a}$ No cases of progressive multifocal leukoencephalopathy were reported

b The event reported in this patient was anaphylactic reaction. No diagnostic tests were performed to confirm or evaluate this event. The patient was transported to the emergency department and observed for $<4 \mathrm{~h}$ but was not hospitalized for evaluation of the event

c The cause of death was reported as "traumatic brain injury, subdural hematoma," and the event was judged by the investigator to be unrelated to treatment. The patient received only one dose of natalizumab. The death was reported $>3$ months after the patient received natalizumab

Several limitations of the STRIVE study, including the lack of a comparator group and the open-label study design, have previously been described [8]. In addition, sample sizes were reduced by attrition and missing data, particularly with respect to the NEDA analyses, which used a very conservative approach with respect to the inclusion of patients. To assess the robustness of the results and address any potential attrition bias, sensitivity analyses on NEDA, Clinical NEDA, MRI NEDA, ARR, CDW, and MRI outcomes were performed based on the data from patients who completed the 4-year study on natalizumab. The results of these sensitivity analyses were similar to those of the ITT population. In addition, reported lack of efficacy was only noted as a reason for treatment discontinuation in a small number of patients, whereas seroconversion to anti-JCV antibody-positive status/elevated index/PML risk was the most common reason for treatment discontinuation, which is consistent with other observational studies that reported anti-JCV antibody-positive status/PML risk as the most common reason for natalizumab discontinuation $[15,17]$. As STRIVE was initiated before the publication of evidence that the anti-JCV antibody level (index) may further differentiate PML risk in natalizumab-treated MS patients without prior immunosuppressant use [6], antiJCV antibody index data were very limited.

While STRIVE was a single-country study, its results are consistent with those of the TOP study, which included 17 countries outside the US. STRIVE, unlike TOP, also provided longterm effectiveness data on MRI outcomes, which are routinely used in clinical practice for monitoring the effectiveness of DMTs. In addition, the STRIVE study population was restricted to patients who were JCV negative at study initiation, which aligns with the JCV antibody status of most patients initiating natalizumab in the US [18]. In STRIVE, $>75 \%$ of patients with available serostatus data at year 4 remained JCV negative.

\section{CONCLUSIONS}

The results from STRIVE support the long-term effectiveness and safety of natalizumab treatment. Identified baseline predictors of NEDA (lower EDSS score and higher T2 lesion volume) 
and Clinical NEDA (higher T2 lesion volume) may be valuable to clinicians in considering the benefits and risks of initiating natalizumab treatment in JCV-negative patients with early RRMS.

\section{ACKNOWLEDGEMENTS}

The authors gratefully acknowledge the STRIVE investigators, listed below, for their efforts and contributions, as well as the patients. STRIVE investigators: Bridget Bagert, $\mathrm{MD}$, Roumen Balabanov, MD, Margaret Burnett, MD, Claudia Chaves, MD, Stanley Cohan, MD, PhD, Joanna Cooper, MD, Eric Eggenberger, DO, John Foley, MD, Edward Fox, MD, PhD, Robert Fox, MD, Dennis Garwacki, MD, Lawrence Goldstick, MD, Benjamin Greenberg, MD, MHS, Mark Gudesblatt, MD, Craig Herrman, MD, Jonathan Howard, MD, John Huddlestone, MD, Mark Janicki, MD, Jeffrey Kaplan, MD, George Katsamakis, MD, Amos Katz, MD, Mariko Kita, MD, Lauren Krupp, MD, Ellen Lathi, MD, Kermit Lloyd, MD, Kenneth Mankowski, DO, Tamara Miller, MD, Stephen Newman, MD, Scott Newsome, DO, Allan Perel, MD, Jai Perumal, MD, John Puente, MD, Marcus Rice, MD, Emily Riser, MD, Peter Riskind, MD, PhD, Teri Schreiner, MD, MPH, Christopher Sheppard, MD, Scott Silliman, MD, Jason Silversteen, DO, Jacob Sloane, MD, PhD, Charles Smith, MD, Ben Thrower, MD, Robert Tillett, MD, Carlo Tornatore, MD.

Funding. Biogen provided funding for this study as well as funding for medical writing support in the development of this manuscript. Biogen also funded the journal's Rapid Service and Open Access Fees for this manuscript.

Authorship. All named authors meet the International Committee of Medical Journal Editors (ICMJE) criteria for authorship for this article, take responsibility for the integrity of the work as a whole, and have given their approval for this version to be published.
Authors' Contributions. Jai Perumal, Roumen Balabanov, Laura Balcer, Steve Galetta, Denise I. Campagnolo, and Robert J. Fox were involved in the conception and design of the study. Ray Su and Roger Chang performed the statistical analyses. All authors were involved in drafting the manuscript, discussing the results, and providing critical revision of the article.

Medical Writing Assistance. Medical writing support for the development of this manuscript, under the direction of the authors, was provided by Alexandra D'Agostino, PhD, of Ashfield MedComms, an Ashfield Health company, and funded by Biogen.

Prior Presentation. Portions of these results were presented at the European Committee for Treatment \& Research in Multiple Sclerosis (ECTRIMS; September 11-13, 2019; Stockholm, Sweden).

Disclosures. Jai Perumal has received fees from Acorda, Biogen, Genzyme, and Teva. Roumen Balabanov has received consulting fees from Biogen, Sanofi, and Teva and grant/research support from Biogen. Ray $\mathrm{Su}$ was an employee of Biogen at the time of these analyses and may hold stock and/or stock options in Biogen; he is now an employee of Inari Medical, which was not involved in this study. Lily Lee was an employee of Biogen at the time of these analyses and may hold stock and/or stock options in Biogen; she is now an employee of TG Therapeutics, which was not involved in this study. Laura Balcer has received consulting fees from Biogen and Genzyme. Steven Galetta has received consulting fees from Biogen. Roger Chang, Denise I. Campagnolo, Robin Avila, and Danette Rutledge are employees of and may hold stock and/or stock options in Biogen. Robert J. Fox has received personal consulting fees from Actelion, Biogen, Celgene, EMD Serono, Genentech, Immunic, Novartis, Sanofi, Teva, and TG Therapeutics; has served on advisory committees for Actelion, Biogen, and Novartis; and has received clinical trial contract and research grant funding from Biogen and Novartis. 
Compliance with Ethics Guidelines. All patients provided written informed consent prior to enrollment. Approval was granted by the Copernicus Group IRB \#1 (reference number IRB00001313) and, at the rest of the study sites, by an independent ethics committee (Supplementary Appendix S1). The study was performed in accordance with the Helsinki Declaration of 1964 and its later amendments.

Data Availability. The datasets generated and/or analyzed during the current study are not publicly available. The authors fully support sharing whenever possible. Requests for deidentified data should be made to Biogen via established company data-sharing policies and processes detailed on the website http:// clinicalresearch.biogen.com/.

Open Access. This article is licensed under a Creative Commons Attribution-NonCommercial 4.0 International License, which permits any non-commercial use, sharing, adaptation, distribution and reproduction in any medium or format, as long as you give appropriate credit to the original author(s) and the source, provide a link to the Creative Commons licence, and indicate if changes were made. The images or other third party material in this article are included in the article's Creative Commons licence, unless indicated otherwise in a credit line to the material. If material is not included in the article's Creative Commons licence and your intended use is not permitted by statutory regulation or exceeds the permitted use, you will need to obtain permission directly from the copyright holder. To view a copy of this licence, visit http:// creativecommons.org/licenses/by-nc/4.0/.

\section{REFERENCES}

1. Polman $\mathrm{CH}, \mathrm{O}^{\prime}$ Connor $\mathrm{PW}$, Havrdova E, et al. A randomized, placebo-controlled trial of natalizumab for relapsing multiple sclerosis. N Engl J Med. 2006;354(9):899-910.

2. Havrdova E, Galetta S, Hutchinson M, et al. Effect of natalizumab on clinical and radiological disease activity in multiple sclerosis: a retrospective analysis of the Natalizumab Safety and Efficacy in Relapsing-Remitting Multiple Sclerosis (AFFIRM) study. Lancet Neurol. 2009;8(3):254-60.

3. Bloomgren G, Richman S, Hotermans C, et al. Risk of natalizumab-associated progressive multifocal leukoencephalopathy. N Engl J Med. 2012;366(20): 1870-80.

4. Ho P-R, Koendgen H, Campbell N, Haddock B, Richman S, Chang I. Risk of natalizumab-associated progressive multifocal leukoencephalopathy in patients with multiple sclerosis: a retrospective analysis of data from four clinical studies. Lancet Neurol. 2017;16(11):925-33.

5. Tysabri (natalizumab) [prescribing information]. Cambridge, MA: Biogen; 2020.

6. Plavina T, Subramanyam M, Bloomgren G, et al. Anti-JC virus antibody levels in serum or plasma further define risk of natalizumab-associated progressive multifocal leukoencephalopathy. Ann Neurol. 2014;76(6):802-12.

7. Polman $\mathrm{CH}$, Reingold SC, Banwell B, et al. Diagnostic criteria for multiple sclerosis: 2010 revisions to the McDonald criteria. Ann Neurol. 2011;69(2): 292-302.

8. Perumal J, Fox RJ, Balabanov R, et al. Outcomes of natalizumab treatment within 3 years of relapsingremitting multiple sclerosis diagnosis: a prespecified 2-year interim analysis of STRIVE. BMC Neurol. 2019;19(1):116.

9. Gorelik L, Lerner M, Bixler S, et al. Anti-JC virus antibodies: implications for PML risk stratification. Ann Neurol. 2010;68(3):295-303.

10. Traboulsee A, Simon JH, Stone L, et al. Revised recommendations of the Consortium of MS Centers Task Force for a standardized MRI protocol and clinical guidelines for the diagnosis and follow-up of multiple sclerosis. AJNR Am J Neuroradiol. 2016;37(3):394-401.

11. Prosperini L, Fanelli F, Pozzilli C. Long-term assessment of no evidence of disease activity with natalizumab in relapsing multiple sclerosis. J Neurol Sci. 2016;364:145-7.

12. Miller DH, Khan OA, Sheremata WA, et al. A controlled trial of natalizumab for relapsing multiple sclerosis. N Engl J Med. 2003;348(1):15-23.

13. Hemond CC, Bakshi R. Magnetic resonance imaging in multiple sclerosis. Cold Spring Harb Perspect Med. 2018;8(5):a028969. 
14. Butzkueven H, Kappos L, Pellegrini F, et al. Efficacy and safety of natalizumab in multiple sclerosis: interim observational programme results. J Neurol Neurosurg Psychiatry. 2014;85(11):1190-7.

15. Butzkueven $H$, Kappos L, Wiendl H, et al. Longterm safety and effectiveness of natalizumab treatment in clinical practice: 10 years of real-world data from the Tysabri Observational Program (TOP). J Neurol Neurosurg Psychiatry. 2020;91(6):660-8.

16. Foley J, Carrillo-Infante C, Smith J, et al. The 5-year Tysabri global observational program in safety (TYGRIS) study confirms the long-term safety profile of natalizumab treatment in multiple sclerosis. Mult Scler Relat Disord. 2019;39:101863.
17. Horakova D, Uher T, Krasensky J, et al. Long-term effectiveness of natalizumab on MRI outcomes and no evidence of disease activity in relapsing-remitting multiple sclerosis patients treated in a Czech Republic real-world setting: a longitudinal, retrospective study. Mult Scler Relat Disord. 2020;46: 102543.

18. Mason L, Campbell N, Ren Z, Chang I, Ho P-R. Low risk of natalizumab-associated progressive multifocal leukoencephalopathy in patients who were anti-JC virus antibody negative at baseline. In: Presented at the 35th Congress of the European Committee for treatment and research in multiple sclerosis; September 11-13, 2019; Stockholm, Sweden. 